Dialectics of Women's Home and Market Work, New York 1980.

Dale Spender (Hrsg.), Men's Studies Modified: The Impact of Feminism on the Academic Disciplines, New York 1981.

Judith Stacey, Patriarchy and the Socialist Revolution in China, Berkeley 1983.

Carol Stack, All Our Kin: Strategies for Survival in a Black Community, New York 1976.

Nany Tanner, Adrienne Zihlman, ,Women in Evolution, Part I: Innovation and Selection in Human Origins“, Signs 1: 585-608, 1976.

Mary Kay Tetreault, „Feminist Phase Theory: An Experience Derived Evaluation Model“, The Journal of Higher education 1985.

E.P. Thompson, The Poverty of Theory and Other Essays, New York 1979

Barrie Thome, „Gender - How it is best conceptual ized?“, Hekt. Ms., Vortrag gehalten auf der Jahresversammlung der American Sociological Association 1978 .

Anna Lowenhaupt Tsing und Sylvia Yunko Yanagisako, „Feminism and Kinship Theory“, Current Anthropology, 1985.

Jill McCalla Vickers, „Memoirs of an Antological Exile: The Methodological Rebellions of Feminist Research",S.27-46in Angela Miles und Geraldine Finn (Hrsg.), Feminism in Canada, Montreal 1982

Lise Vogel, Marxism and the Oppression of Women, New Brunswick 1984.

Patricia Voydenoff (Hrsg.), Work and Family, Palo Alto 1983.

Beverly M. Walker, „Psychology and Feminism - if you can't beat them, join them!, S. 111-124 in Dale Spender (Hrsg.), Men's Studies Modified, New York 1981.

Immanuel Wallerstein, The Capitalist World Economy,New York 1979.

Leonore J. Weitzman, The Marriage Contract, New York 1981.

Candace West und Don Zimmerman, „Small Insults: A Study of Interruption in Cross-sex Conservations between Unacquainted Persons", S. 103-118 in Barrie Thorne, Cheris Kramarae und Nancy Henley (Hrsg.), Language, Gender and Society, Rowely, Mass., 1983.

Ann Whitehead, „Sexual Antagonism in Herefordshire", S. 169-203 in Diana L. Barker und Shiele Allen (Hrsg.), Dependance and Explcitation in Work and Marriage, London 1976.

Jery Dawn Wine, „Gynocentric Values and Feminist Psychology“, S. 67-88 in: Angela Miles und Geraldine Finn (Hrsg.) Feminism in Canada, Montreal 1982.

Eric Wolf, Europe and the People Without History, Berkeley 1982.

Erik Olin Wright, Cynthia Costello, Dabid Hachen und Joey Sprague, "The American Class Structure", American Sociological Review 47: 709-726, 1982.
Maxine Baca Zinn, "Mexican Women in the Social Sciences $^{4}$, Signs 8: 259-272, 1982.

\section{Renate Bridenthal}

\section{Marxistisch- feministische Ansätze zur Frauengeschichte in den USA}

Vor einigen Jahren prägten die fünf marxistischfeministischen Gruppen - jede mit ca. 30 Mitgliedern - , die Anfang der 70er Jahre entstanden waren, die feministische Diskussion an der Ostküste nachhaltig. Im März 1977 trafen wir uns zu einer Arbeitstagung, auf der versucht wurde, die Arbeit der vergangenen Jahre zusammenzufassen und politische Strategien zu diskutieren.

Ich erinnere mich an die hoffnungsvolle, aber leicht unbehagliche Atmosphäre im Raum als wir unser Selbstverständnis zu formulieren versuchten. Diese Unbehaglichkeit wurde von Mary Bailey, Sprecherin von „MF 2“, auf den Punkt gebracht:

Als marxistisch-feministische Frauen sitzen wir ritlings auf einem widerspenstigen Pferd. Wir sind uns eigentlich über diesen Bindestrich noch nicht im Klaren. Wir glauben, daß es eine Revolution ist, die von den Erkenntnisses des Feminismus wie des Marxismus ausgeht - daß es das ist, was wir wollen (. . .) Aber leider hat es allzu oft nicht mehr bedeutet, als daß wir „Marxisten" für unsere feministischen Schwestern und „Feministinnen" für unsere marxistischen Brüder sind. Die größte Gefahr, der wir uns im Augenblick gegenübersehen, ist, daß wir uns mit diesem Bindestrich bequem einrichten, als Ersatz für eine Selbsidefinition . . Bindestrich als Wunschvorstellung. Wir haben seine Zeichen an der Wand gesehen.

Die Politologin Rosalynn Petchesky wies auf vier wichtige Bereiche hin, die wir als marxistische Feministinnen zu untersuchen begonnen hatten: 1. Das Verhältnis zwischen Familie (bzw. Verwandtschaftssystemen) und der Klassenstruktur einer Gesellschaft. 2. Das Verhältnis von Kontrolle über die Reproduktionsmittel 
und Macht der Mãnner. 3. Die Beziehung zwischen inännlicher Vorherrschaft und Staat. 4. Der Zusammenhang zwischen diesen drei Relationen und sein Bezug zum BewuBtsein von Frauen; und hier die Frage: was ist revolutionäre Transformation?' Diese Thematik, wurde in den folgenden Jahren in weiteren, größeren Arbeiten behandelt, die ich hier zusammenfassend darstellen möchte.

Zum Schwerpunkt: Verhältnis zwischen Familie und Klassenstruktur, hat die Anthropologin Rayna Rapp vorgeschlagen, zwischen den Kategorien Familie und Haushalt genau zu unterscheiden. Der Haushalt sei die eigentliche Arbeitsgemeinschaft, in der Güter und Aufgaben verteilt werden, die wiederum mit anderen gesellschaftlichen Bereichen vermittelt sind und zur Reproduktion der Gesellschaft beitragen. „Familie“ ist dagegen „nur ein Konzept, welches definiert, wer im Haushalt leben soll." In unserer Zivilisation funktionieren Familien wie Rekrutierungsagenturen des Haushalts, dessen Funktion und Struktur wiederum klassenspezifisch differenziert werden muB. Bäuerliche, proletarische, bürgerliche und aristokratische Familien unterscheiden sich voneinander, indem ihre Haushalte verschiedene Formen gesellschaftlicher Mitwirkung ausüben. Man wird in einen Haushalt hineingeboren und zählt deshalb zu einer bestimmten Familie.

Die Bedeutung von Rapps Ansatz liegt in der Modifizierung des Familienkonzeptes, unter dem Frauen bisher begrifflich begraben worden sind. An seine Stelle tritt der Haushalt als gesellschaftliche Einheit aus ökonomischem Eigeninteresse handelnder Individuen. ${ }^{2}$ Die Historikerin Mary Ryan hat eine interessante Arbeit über die Familie als Wiege des Bürgertums vorgelegt: Cradle of the Middle Class: The Family in Oneida County, New York 1780-1865 (Cambridge, 1981), in der Rapps Annahmen empirisch belegt werden. Ryan zeigt, wie wechselnde Wirtschaftslagen im frühen 19. Jahrhundert Veränderungen der Familienformen und innerfamiliären Verhältnisse bedingten. Sie zeigt, wie sich Oneida von einer Agrargesellschaft, deren Hauswirtschaften die erweiterten Familien zusammenschlossen, zu einer Handels- und Industriegesellschaft wandelte, in der außerhalb des Hauses produziert wurde und dem verkleinerten Haushalt andere Aufgaben wie Konsum, Reproduktion und emotionale Stützung zugewiesen wurden. Die neuen Familienverhältnisse zeigten weniger patriachalische Gewalt, da es nun an văterlicher Kontrolle über die Produk- tionsmittel fehlte, dagegen größere Verehrung von Mütterlichkeit und stärkere Berücksichtigung der besonderen Bedürfnisse von Kindern. Dieser Wandel der Beziehungen innerhalb der Familie basierte auf der Veränderung der Subsistenzökonomie in eine Produktionsweise des Tausches und des Profits. Der Wandel wurde jedoch von Einzelnen gestaltet, die "ihre eigene Geschichte machten ", wie Marx formulierte.

Dieser Übergang von einer Familienform zur anderen war vermittelt durch karitative und sozialreformerische Frauenvereine, in denen man nicht-verwandtschaftliche Beziehungen $2 \mathrm{u} \mathrm{Al}$ ters- und Geschlechtsgenossinnen anknüpfte. Da diese Vereine besonders für neu eingewanderte junge Leute familienartige, emotionale Dienste anboten, wurden sie zu einem Vermittlungsinstrument neuer häuslicher Werte, die allerdings nun durch egalitäre Vorstellungen modifiziert waren. Nachdem die Neuankömmlinge in die Gesellschaft Oneidas integriert waren und die ökonomische Anpassungskrise überwunden war, lösten sich die Verbände auf und die aufstrebende Mittelklasse kehrte zum Familienverband als Primärorganisation zurück. Ein neuer Kult der Häuslichkeit glorifizierte das Private im Gegensatz zu öffentlichen Vereinigungen und verbannte Frauen an den häuslichen Herd, um die Kinder für den gesellschaftlichen Aufstieg zu erziehen. Ryan zeigt den Widerspruch auf, daB Frauen öffentliche Ämter bekleiden, um private Rollen zu schützen; zum Beispiel in Kampagnen für öffentliche Moral und gegen das Laster, um die häuslichen Tugenden zu wahren. Auf diese Weise schufen sie eine Sphäre, in der sie innerhalb der Gesellschaft Macht ausüben konnten. Allerdings besteht ein zweiter Widerspruch darin, daB diese Sphäre sehr bald zum Ghetto wurde, auf das ihre öffentlichen Aktivitäten beschränkt wurden, wogegen spätere Frauengenerationen schlie $B-$ lich rebellierten. Der Widerspruch, daß Familien sich durch den EinfluB von nicht zur Familie gehörigen Altersgenossen auflösten, um sich dann wieder neu zu formieren, erklärt einen Prozeß, den man bisher zwar theoretisch selbstverständlich angenommen hat: die Privatisierung der Reproduktion bei gleichzeitiger Vergesellschaftung der Produktion als historisch konkrete Handlungsmuster bestimmter Personengruppen.

Ryans Buch ist ein gutes Beispiel für einen methodologischen Ansatz, den die Historikerin Joan Kelly 1976 vorstellte: 
die historischen Formen, die das Patriarchat annimmt, müssen auf die gesellschaftlichen Produktionsformen zurückgefühn werden. Die Hierarchie zwischen den Geschlechtern verändert sich mit der allgemeinen Organisation von Besitz und Arbei, weil sie sowohl den privaten und öffentlichen Bereich gestaltet und festlegt, inwieweil diese sich einander annähern oder auseinanderfallen. ${ }^{3}$

Ryan zeigt, daB sich der private und der öffentliche Bereich zunāchst durch freiwillige Verbände gegenseitig annähern, um späterhin auseinanderzufallen, wobei die Ursache als ein Wandel in der allgemeinen Organisation von Besitz und Arbeit dargestellt wird.

Auch andere marxistisch-feministische $\mathrm{Hi}$ storikerinnen haben auf mögliche Interessengegensätze innerhalb der Familien hingewiesen. Da ein Großteil der traditionellen marxistischer. Geschichtsschreibung dazu tendiert, die proletarische Familie als sicheren Hort in Klassenkonflikten zu sehen, hat dieser feministische Anschlag auf die monolithische Vorstellung von Familie einige Aufregung verursacht, und dazu geführt, daB dem Geschlechterkonflikt innerhalb des Proletariats mittlerweile eine größere Bedeutung beigemessen wird. Die Beispiele lassen sich vermehren: Geburtenkontrolle, meist als friedliche Familienentscheidung dargestellt, war in Wirklichkeit nicht nur in der öffentlichen Debatte umstritten sondern auch Gegenstand häuslicher Konflikte. Ebenso waren Migrationen wie der „Zug nach Westen“ nicht nur Unternehmungen mit voller Zustimmung aller Familienmitglieder. Viele Frauen sträubten sich bitterlich gegen ihre Entwurzelung. Weiterhin zeigt die Untersuchung von Haushaltsplänen, $\mathrm{daB}$ es nicht einen sondern mehrere Lebensstandards in einem Haushalt gegeben hat: Der Vater bekam meistens mehr Fleisch, Taschengeld, Freizeit, usw. ${ }^{4}$

Neuere Studien über häusliche Gewalt zeigen, daß Extreme von familiärer Brutalität und sogar Mord durchaus nicht unüblich sind, wobei zumeist Frauen und Kinder die Opfer sind:

Feministisches Umdenken hat das Haus nicht nur als einen Arbeitsplatz entmystifiziert, sondern auch als einen $O r$ intensiver Intimität und Begegnungen von nicht unbedingt liebevoller Art. Es hat das GrußkartenImage der lächelnden Familie geröngt und dabei das Zähnefletschen von Wut und Leid enthüllt: Fälle von Inzest, Vergewaltigung, Prügel, Seelenmord - und nicht vereinzelt, sondern als ein erstaunlich verbreitetes Phänomen. Die Familie ist nach klischeehafien positiven Darstellungen demaskiert worden . . . Familienharmonie existiert, aber sie ist eine Errungenschaft, kein allgegenwärtiger, naturgegebener Zustand. ${ }^{5}$
Über die Bedeutung von Hausarbeit in Bezug auf die Gesamtökonomie ist in den letzten Jahren eine lebhafte Diskussion geführt worden. Eine der Fragen, die sich in diesem Zusammenhang stellt: Wie wirkt sich das Wechselverhältnis zwischen unbezahlter und bezahlter Arbeit auf den Zusammenhalt der Arbeiterklasse aus? Gewinnen oder verlieren Männer der Arbeiterklasse eher durch den Status der Hausfrauen als Reserve des Arbeitsmarktes? Es ist darauf hingewiesen worden, daB das ,Familieneinkom: men', das historisch durch organisierte Arbeitskämpfe durchgesetzt worden ist, nur einigen wenigen Männern und Frauen der Arbeiterklasse zugute gekommen ist. Ungelernte Arbeiter und die meisten Frauen gingen leer aus, einschließlich weiblicher Familienvorstände. Als billige Arbeitskräfte jedoch stellten Frauen eine Bedrohung für gelernte Arbeiter dar, wenn in Phasen technologischer Entwicklungsschübe Frauen bevorzugt eingestellt wurden, um die Empfänger des höheren ,Familieneinkommens' zu ersetzen. Die erste Reaktion männlicher Arbeiter war ein entschiedener Widerstand gegen die Einstellung von Frauen; dies ist zweifelsohne kein Akt von Klassensolidarität. ${ }^{6}$ Die folgenden Reaktionen waren sehr viel subtiler. Dazu gehörten von der organisierten Arbeiterschaft durchgesetzte Schutzbestimmungen, die zunächst den Anschein erweckten, als würden sie die Arbeitsbedingungen für Frauen menschlicher gestalten. Sie sorgten aber zur gleichen Zeit dafür, daß Frauen von vielen Arten einträglicher Berufe ausgeschlossen wurden.?

Andererseits hat die historische Frauenforschung auch Beispiele von Solidarität zwischen Männern und Frauen aufgefunden. Carol Turbin schreibt über das Bündnis der Waschfrauen mit den männlichen Eisengießem im 19. Jahrhundert in Troy, New York, wenn es bei einer der beiden Gruppen Streik gab. Sie zeigt, wie Familienbindungen zwischen Arbeitern in diesen Berufen politische Bündnisse ermöglichten. ${ }^{8}$ Ruth Milkman berichtet von den Arbeitern in den Autofabriken während des zweiten Weltkriegs. Nachdem sie sich zuerst gegen Frauenarbeit gesträubt hatten, um ihre Arbeit nicht durch niedrigere Frauenlöhne entwerten zu lassen, setzten sie sich doch für gleichen Lohn für die Arbeiterinnen ein. ${ }^{9}$

Eine der wichtigsten Beiträge feministischer Forschung ist die Erkenntnis, daß Frauen Handelnde in der Geschichte sind, die die Welt in ihrem eigenen Interesse mitgestalten. Was hier marxistische von anderen Feministinnen unter- 
scheidet, ist die Analyse des Verhältnisses zwischen Klasse und Geschlecht und zwischen Frauenbewegungen und anderen sozialen Bewegungen. Es haben über die Klassen hinweg Bündnisse von Frauen zu unterschiedlichen Zwecken, zur Frauenbefreiung, für die Organisation von Arbeit und für Geburtenkontrolle bestanden. So hat Meredith Tax in ihrem Buch, The Rising of the Women: Feminist Solidarity and Class Conflict, 1880-1917 (Monthly Review Press, New York 1980) gezeigt, daß es in den U.S.A. einer Einheitsfront von Frauen aus der Arbeiterbewegung, der sozialistischen Partei und der bürgerlichen Frauenbewegung des 19. Jahrhunderts zu verdanken ist, daß sich um 1880 einige neue Gesetze in Illinois zugunsten der Arbeiterfrauen durchsetzen ließen. Mit einsetzender ökonomischer Krise wurden die genannten Organisationen geschwächt, die Front fiel auseinander und manches Erkämpfte wurde eingebüßt. Ähnlich beschreiben auch die englischen marxistischen Feministinnen Jill Liddington und Jill Norris die Verhältnisse in ihrem Buch One Hand Tied Behind Us: The Rise of the Women's Suffrage Movement (Virago: London, 1978). Sie zeigen die Brüchigkeit von Bündnissen zwischen Frauen der Mittelklasse und Arbeiterinnen im Kampf um das Wahlrecht in England auf. Judith Walkowitz beschreibt ähnliche Probleme im Zusammenhang mit dem Kampf von Frauen um freie Sexualität in ihrem Buch Prostitution and Victorian Society: Women, Class and the State (Cambridge University Press: 1980). In dem Kampf um die Zurücknahme des "Contagious Diseases Act“ (Gesetz zur Verhütung ansteckender Krankheiten) in England während der 70er Jahre des letzten Jahrhunderts argumentierten die Gegnerinnen, daB erzwungene medizinische Untersuchungen von Prostituierten eine Beleidigung für alle Frauen seien, weil ausschlieBlich Frauen für die Verbreitung von Geschlechtskrankheiten verantwortlich gemacht würden und der Polizei große Freiheiten für mögliche Untersuchungen verdächtiger Frauen einräume. Ihre Bemühungen zahlten sich zunächst aus, allerdings wurden die alten Gesetze späterhin durch neue, verschärfte Gesetze ersetzt, die die Mittelstandsfrauen schließlich zu Komplizinnen bei der Verfolgung derer werden liessen, die sie einst ihre Schwestern genannt hatten. In Linda Gordons Buch Woman's Body, Woman's Right: A Social History of Birth Control in America (Grossmann Publishers, Viking Press: New York, 1976) wird der jahrhundertelange Kampf um die Gebur- tenkontrolle von feministischen Ansprüchen bis zu einer imperialistisch kontrollierten Bevölkerungspolitik nachgezeichnet. Der Umschwung ins Gegenteil wurde durch eine von Margaret Sanger initiierte Strategie des Kompromisses mit wohlsituierten Sympathisantinnen und der etablierten Ärzteschaft herbeigeführt. Individuelle weibliche Kontrolle wurde der männlichen Kontrolle durch Ärzte übertragen und schließlich der staatlichen Intervention untergeordnet, wie im Falle unfreiwilliger Sterilisation. Hier war ein Bündnis zwischen den Klassen offensichtlich fehlgeschlagen. Sanger selbst verkörperte den Wandel von einer Sozialistin und Feministin zu einer Sozialdarwinistin am Rande des Faschismus.

In England haben sich die beiden marxistischen Feministinnen Mary McIntosh ${ }^{10}$ und Eljzabeth Wilson mit dem Verhältnis zwischen Klassen- und Männerherrschaft auseinandergesetzt.

Wilson vertritt in ihrem Buch Women and the Welfare State (Tavistock Publications, London 1977) die Auffassung, daB die Einrichtung der sozialen Wohlfahrt Ambivalenzen schafft, indem sie die Lebensqualität zwar einerseits verbessert, während sie sie gleichzeitig zunichtemacht. Diese Einrichtung wurde von den Arbeitern erkämpft, um die schlimmsten kapitalistischen Auswüchse zu mildern, jedoch wird Sozialpolitik auch als Instrument der Regierungen versuchen, eine bestimmte Gesellschaftform herbeizuführen, die das Wachstum des Kapitalismús fördert. Wilson argumentiert dessen ungeachtet, daß der Widerstand gegen die Kürzungen der Sozialleistungen ein wesentlicher Bestandteil des Klassenkampfes in der Staatsarena sei. Ihrer Meinung nach müssen Frauen besonders die einseitig staatliche Definition von Frauen als Mütter in Frage stellen und auf weiteren Forderungen beharren.

Die umstrittene Frage des Bindestriches zwischen Marxismus und Feminismus wurde vor kurzem von Lise Vogel besprochen in ihrem Buch Marxism and the Oppression of Women: Toward a Unitary Theory (Rutgers University Press, 1983). Der erste Teil des Buches gibt einen Überblick über die Schriften von Marx und Engels selber, und zeichnet die Entwicklung ihrer eher implizit bleibenden Theorie über Frauenemanzipation nach.

Einerseits findet Vogel, daB Marx und Engels die Vorurteile ihrer Zeit nicht überwunden hatten und dadurch immer wieder eine ,natürliche" (und nicht historische) Arbeitsteilung in 
der Familie unterstellen. Andererseits findet Vogel, daB die Schriften doch eine Basis für wirkliche Frauenemanzipation anbieten mit ihrer Einsicht, daB Individuum und Familie nicht zeitlose Abstraktionen sind, sondern sich mit den historischen Formen der Eigentumsverhältnisse verändern. Außerdem, sagt Vogel, ist anzuerkennen, daB Marx und Engels sich praktisch in dieser Richtung bemühten, indem sie sich in der Ersten Internationalen gegen die Gewerkschafter und Anarchisten sträubten, welche die Frau vom Arbeitsmarkt verdrängen wollten und für die Aufnahme der Frauen in die Gewerkschaften plädierten.

Andererseits kritisiert Vogel an Engels, daß er unentschieden bleibt zwischen der Konstruktion einer gesellschaftlich „naturwüchsig“ produzierten Familie und geschlechtlicher Arbeitsteilung und andererseit der bewußt und unbewußt vertretenen Position ihrer Natürlichkeit. Damit führe er einen fatalen Dualismus ein, in dem weder kapitalistische noch patriarchalische Strukturen einen Anknüpfungspunkt finden, und damit jede Dialektik der Entwicklung verloren geht. Um diesen fatalen Dualismus haben heutige ,sozialistische Feministinnen“ Engels beerbt, zum Schaden einer klaren Theorie.

Aus dem Amerikanischen übersetzt von Renate Bridenthal und Renate Steinchen

\section{Anmerkungen}

1 Rosalind Petchesky, ..Dissolving the Hyphen: A Report on Marxist-Feminist Groups $1-5^{\prime 4}$, in Zillah R. Eisenstein, Capitalist Patriarchy and the Case for Socialist Feminism, New York and London, 1979, S. 375.

2 Rayna Rapp, „Family and Class in Contemporary America: Notes Roward an Understanding of Ideology", Science and Society, vol. XLII, no. 3, 1978, S. 278-300. Auch Rayna Rapp, Ellen Ross, und Renate Bridenthal, .Examining Family History", Feminist Studies, vol. 5, no. 1, 1979, S. 174-200.

3 Joan Kelly-Gadol, „The Social Relation of the Sexes: Methodological Implications of Women's History“, SIGNS, vol. 1, no. 4, 1976, S. 821.

4 Rapp, Ross und Bridenthal in Feminist Studies.

5 Renate Bridenthal, „The Family: The View from a Room of Her Own", Rethinking the Family: Some Feminist Questions, hrsg. von Barrie Thorne, New York und London 1982, S. 234.

$6 \mathrm{Zu}$ dieser Frage existiert eine große Anzahl von $\mathrm{Li}$ teratur. $\mathrm{Zu}$ den interessantesten Arbeiten gehören: Jane Humphries, „Class Struggle and the Per- sistence of the Working Class Family", Cambridge Joumal of Economics 1:3, 1977 a; Humphries, .The Working Class Family, Women's Liberation and Class Struggle: The Case of Nineteenth Century British History“. Review of Radical Political Economics 9:1, 1977; Heidi Hartmann, „Capitalism, Patriarchy and Job Segregation by Sex", SIGNS, vol. 1, no. 3 Teil 2, 1976. Nachdruck in Eisenstein, op. cit., S. 206-247; Gita Sen, „The Sexal Division of Labor and the Working-Class Family: Towards a Conceptual Synthesis of Class Relations and the Subordination of Women", $R e$ view of Radical Political Economics, 12:2. 1980. S. $76-86$.

7 Siehe die Sonderausgabe über Frauen und Schutzbestimmungen von Feminist Studies, vol. 5, no. 2, 1979 und Judith Baer, The Chains of Protection. Judicial Response to Woman's Labor Legislation, Westport/Conn., 1978.

8 Carole Turbin, "And We Are Nothing But Women: Irish Working Women in Troy", in: Women of America: A History, hrsg. von Carol Ruth Berkin and Mary Beth Norton Boston, 1979, S. 202-222.

9 Ruth Milkman, „Redefining ,Women's Work': The Sexual Division of Labor in the Auto Industry During World War II“", Feminist Studies, vol. 8, no. 2,1982 , S. 337-372.

10 Mary McIntosh, „The State and the Oppression of Women", in Feminism and Materialism: Women and Modes of Production, hrsg. von Annette Kuhn und Annemarie Wolpe, London 1978, S. 254-289.

\section{Geneviève Fraisse}

\section{Feministische Singularität - Kritische Historiographie der Geschichte des Feminismus in Frankreich $^{1}$}

Es gibt eine Besonderheit der französischen Geschichte des Feminismus: Sie ist am Schnittpunkt von Sozialgeschichte und Geistesgeschichte (histoire des mentalités) entstanden und gehört doch meines Erachtens weder der ei- 